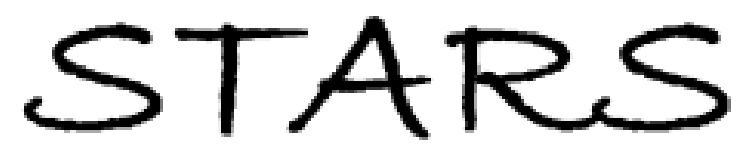

University of Central Florida

STARS

$1-1-2004$

\title{
Extended Cauchy equations for the refractive indices of liquid crystals
}

Jun Li

University of Central Florida

Shin-Tson Wu

University of Central Florida

Find similar works at: https://stars.library.ucf.edu/facultybib2000 University of Central Florida Libraries http://library.ucf.edu

This Article is brought to you for free and open access by the Faculty Bibliography at STARS. It has been accepted for inclusion in Faculty Bibliography 2000 s by an authorized administrator of STARS. For more information, please contact STARS@ucf.edu.

\section{Recommended Citation}

$\mathrm{Li}$, Jun and Wu, Shin-Tson, "Extended Cauchy equations for the refractive indices of liquid crystals" (2004). Faculty Bibliography 2000s. 4538.

https://stars.library.ucf.edu/facultybib2000/4538

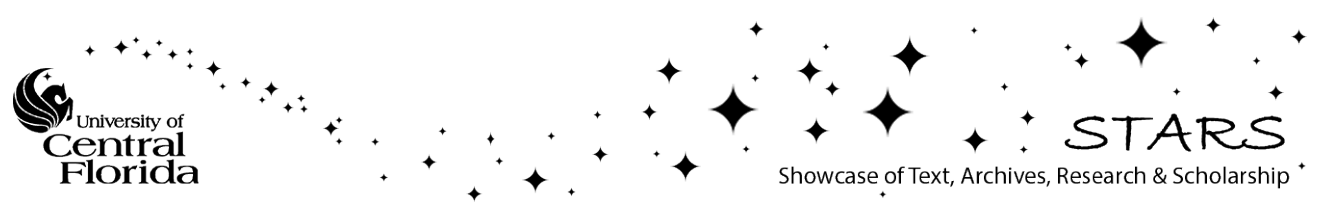




\section{Extended Cauchy equations for the refractive indices of liquid crystals}

Cite as: Journal of Applied Physics 95, 896 (2004); https://doi.org/10.1063/1.1635971

Submitted: 08 September 2003 . Accepted: 29 October 2003 . Published Online: 20 January 2004

Jun Li, and Shin-Tson Wu

\section{ARTICLES YOU MAY BE INTERESTED IN}

Temperature effect on liquid crystal refractive indices

Journal of Applied Physics 96, 19 (2004); https://doi.org/10.1063/1.1757034

Infrared refractive indices of liquid crystals

Journal of Applied Physics 97, 073501 (2005); https://doi.org/10.1063/1.1877815

Two-coefficient Cauchy model for low birefringence liquid crystals

Journal of Applied Physics 96, 170 (2004); https://doi.org/10.1063/1.1738526

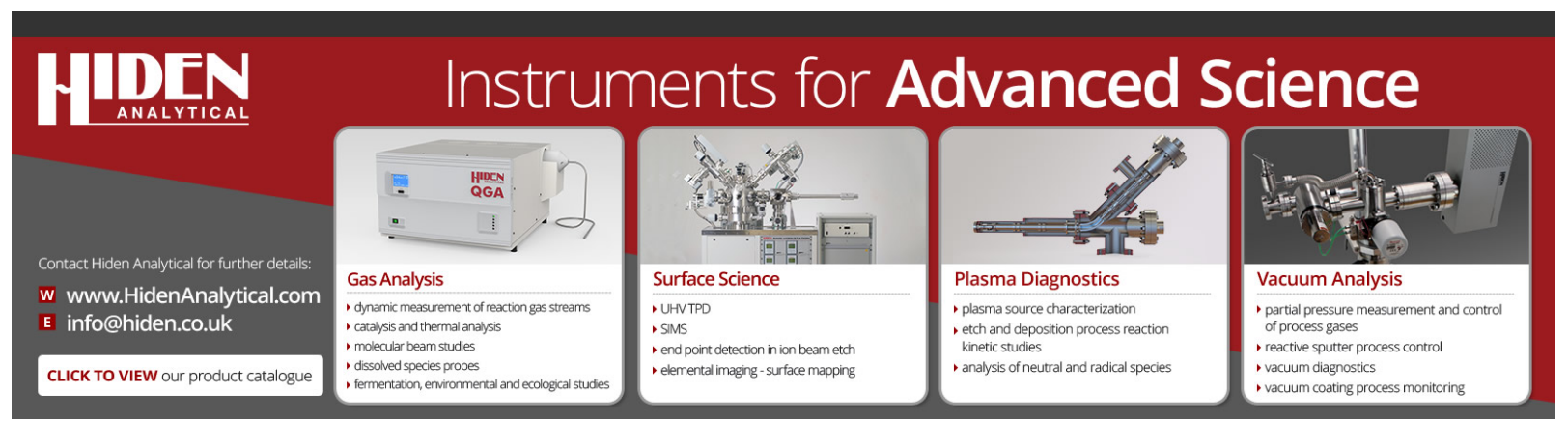




\title{
Extended Cauchy equations for the refractive indices of liquid crystals
}

\author{
Jun $\mathrm{Li}$ and Shin-Tson $\mathrm{Wu}^{\mathrm{a})}$ \\ School of Optics/CREOL, University of Central Florida, Orlando, Florida 32816
}

(Received 8 September 2003; accepted 29 October 2003)

\begin{abstract}
The extended Cauchy equations are derived based on the Vuks equation for describing the wavelength- and temperature-dependent refractive indices of liquid crystal compounds and mixtures. This model fits experimental data well in the off-resonance spectral region. Correlations between the Cauchy coefficients for the nematic and isotropic phases are derived and validated by experiments. (C) 2004 American Institute of Physics. [DOI: 10.1063/1.1635971]
\end{abstract}

\section{INTRODUCTION}

Refractive indices of a liquid crystal (LC) are fundamentally interesting and practically useful parameters. Most liquid crystal light modulators, e.g., flat panel display devices, utilize the electric-field-induced refractive index change. In addition to the molecular constituents, the wavelength and temperature are the two most important factors affecting the LC refractive indices. For instance, to achieve a full-color display three primary colors [red, green, and blue (RGB)] are used. It is essential to know the wavelength-dependent refractive indices of the LC mixture employed in order to optimize the cell design. As the operating temperature changes, the refractive indices change accordingly. The temperature effect is particularly important for projection displays. Due to the thermal effect of the lamp, the temperature of the display panel could reach $50-60{ }^{\circ} \mathrm{C}$. It is important to know the LC properties at the anticipated operating temperature beforehand.

Liquid crystal is a complex molecular system involving short and long range molecular interactions. Several models have attempted to address the wavelength and temperature dependencies of the LC refractive indices. ${ }^{1-9}$ Each one has its own merits and demerits. The Vuks model ${ }^{2}$ is analogous to the classical Clausius-Mossotti equation for correlating the microscopic LC molecular polarizability to the macroscopic refractive indices. However, the wavelength and temperature effects are not described explicitly. The single band model $^{4}$ gives an explicit expression on the wavelength and temperature dependence for birefringence, but not for the individual refractive indices. On the other hand, the threeband model ${ }^{7}$ describes the origins of the LC refractive indices for single LC compounds. Unfortunately, the three-band model needs three fitting parameters for each LC compound. If a LC mixture consists of 5-10 distinct molecular structures, it would be too complicated for the three-band model to quantitatively describe the LC refractive indices of the mixture.

In this article, we derive the extended Cauchy equations for describing the wavelength- and temperature-dependent refractive indices of anisotropic liquid crystals. The original Cauchy equation was intended for the isotropic gases and

\footnotetext{
${ }^{\text {a)} E l e c t r o n i c ~ m a i l: ~ s w u @ m a i l . u c f . e d u ~}$
}

liquids. ${ }^{10}$ The extended Cauchy equations not only apply to single compounds but also to LC mixtures. In Sec. II, we briefly discuss the pros and cons of the three-band model and show the derivation processes for the extended Cauchy equations. In Sec. III, we validate the extended Cauchy equations by experimental data. Excellent agreement between theory and experiment is obtained.

\section{THEORY}

In this section, we give analytical expressions of the three-band model and the extended Cauchy model and discuss their pros and cons.

\section{A. Three-band model}

In the three-band model, ${ }^{7}$ one $\sigma \rightarrow \sigma^{*}$ transition (designated as the $\lambda_{0}$ band) and two $\pi \rightarrow \pi^{*}$ transitions (designated as the $\lambda_{1}$ and $\lambda_{2}$ bands with $\lambda_{2}>\lambda_{1}$ ) are considered. The $\lambda_{0}$ band is located in the vacuum UV region $\left(\lambda_{0}\right.$ $\sim 120 \mathrm{~nm}$ ), and $\lambda_{1}$ is located between 190 and $210 \mathrm{~nm}$, not too sensitive to the LC structure, and $\lambda_{2}$ increases substantially as the molecular conjugation increases. For example, for the 4-cyano-4- $n$-pentyle-cyclohexane-phenyl (5PCH) LC compound, its $\lambda_{1}=200 \mathrm{~nm}$ and $\lambda_{2}=235 \mathrm{~nm}$ while for the 4-cyano-4- $n$-pentylbiphenyl (5CB) its $\lambda_{1}$ shifts to $210 \mathrm{~nm}$ and $\lambda_{2}$ shifts to $282 \mathrm{~nm}^{8}$. This is because 5CB has a longer molecular conjugation than $5 \mathrm{PCH}$.

In the three-band model, the refractive indices $\left(n_{e}\right.$ and $n_{o}$ ) are expressed as follows: ${ }^{7}$

$$
\begin{aligned}
& n_{e} \cong 1+g_{0 e} \frac{\lambda^{2} \lambda_{0}^{2}}{\lambda^{2}-\lambda_{0}^{2}}+g_{1 e} \frac{\lambda^{2} \lambda_{1}^{2}}{\lambda^{2}-\lambda_{1}^{2}}+g_{2 e} \frac{\lambda^{2} \lambda_{2}^{2}}{\lambda^{2}-\lambda_{2}^{2}}, \\
& n_{o} \cong 1+g_{0 o} \frac{\lambda^{2} \lambda_{0}^{2}}{\lambda^{2}-\lambda_{0}^{2}}+g_{1 o} \frac{\lambda^{2} \lambda_{1}^{2}}{\lambda^{2}-\lambda_{1}^{2}}+g_{2 o} \frac{\lambda^{2} \lambda_{2}^{2}}{\lambda^{2}-\lambda_{2}^{2}} .
\end{aligned}
$$

In the visible and infrared regions where $\lambda \gg \lambda_{0}$, the $\lambda_{0}$ band can be approximated by a constant and Eqs. (1a) and (1b) are simplified as

$$
\begin{aligned}
& n_{e} \cong 1+n_{0 e}+g_{1 e} \frac{\lambda^{2} \lambda_{1}^{2}}{\lambda^{2}-\lambda_{1}^{2}}+g_{2 e} \frac{\lambda^{2} \lambda_{2}^{2}}{\lambda^{2}-\lambda_{2}^{2}}, \\
& n_{o} \cong 1+n_{0 o}+g_{1 o} \frac{\lambda^{2} \lambda_{1}^{2}}{\lambda^{2}-\lambda_{1}^{2}}+g_{2 o} \frac{\lambda^{2} \lambda_{2}^{2}}{\lambda^{2}-\lambda_{2}^{2}} .
\end{aligned}
$$


In Eq. (2), $\lambda_{1}$ and $\lambda_{2}$ could be determined from the ultraviolet absorption spectra of the compounds studied. The three unknowns are $n_{0 e}, g_{1 e}$, and $g_{2 e}$ for $n_{e}$ and $n_{0 o}, g_{1 o}$, and $g_{2 o}$ for $n_{o}$. The major advantages of the three-band model are twofold: (1) it explains clearly the origins of the LC refractive indices, and (2) it allows quantitative assessment of each band's contribution to the overall refractive indices. ${ }^{5}$ However, as shown in Eqs. (2a) and (2b) there are three unknowns involved in each index. For single LC compounds, this model works well. However, a LC mixture often consists of 5-10 components with different structures in order to lower the melting temperature and widen the nematic range. If each compound has its own three independent parameters, then a mixture would have too many unknowns. Thus, it is impractical for the three-band model to accurately describe the refractive indices of a complicated LC mixture unless the individual molecules all have similar core structures.

\section{B. Extended Cauchy equations}

The original Cauchy equation is intended for isotropic liquids and gases. Several authors have attempted to fit the refractive indices of anisotropic liquid crystals using Cauchy equations. ${ }^{11,12}$ Although the fitting results are still satisfactory, the physical meaning is not clear.

In this section, we derive the extended Cauchy equations starting from the Vuks equation, which correlates the microscopic molecular polarizabilities $\left(\alpha_{e, o}\right)$ of a liquid crystal to the macroscopic refractive indices: ${ }^{2}$

$$
\frac{n_{e, o}^{2}-1}{\left\langle n^{2}\right\rangle+2}=\frac{4 \pi}{3} N \alpha_{e, o} .
$$

In Eq. (3), $n_{e}$ and $n_{o}$ are refractive indices for the extraordinary and ordinary rays, respectively, $N$ is the number of molecules per unit volume, $\alpha_{e, o}$ is the molecular polarizability, and $\left\langle n^{2}\right\rangle$ is the average value of the refractive indices defined as

$$
\left\langle n^{2}\right\rangle=\left(n_{e}^{2}+2 n_{o}^{2}\right) / 3 .
$$

In the Vuks equation, the local field effect is assumed to be isotropic. The Vuks equation provides some important physical insights for understanding the refractive indices of an anisotropic liquid crystal. However, its descriptions on the wavelength and temperature effects for $n_{e}$ and $n_{o}$ are not explicit.

By substituting Eq. (4) into Eq. (3) and separating $n_{e}$ and $n_{o}$, we obtain

$$
\begin{aligned}
& n_{e}=\left[1+\frac{4 \pi N \alpha_{e}}{1-\frac{4}{9} \pi N\left(2 \alpha_{o}+\alpha_{e}\right)}\right]^{1 / 2}, \\
& n_{o}=\left[1+\frac{4 \pi N \alpha_{o}}{1-\frac{4}{9} \pi N\left(2 \alpha_{o}+\alpha_{e}\right)}\right]^{1 / 2} .
\end{aligned}
$$

Equations (5a) and (5b) are very similar except for the different $\alpha$. For most liquid crystals developed so far, their refractive indices are around 1.45-1.80. Therefore, the second term in the square root is larger than 1, but less than 2.24. In order to expand Eq. (5) into a power series, we rewrite Eq. (5a) as

$$
n_{e}=\sqrt{2}\left[1+\left(\frac{2 \pi N \alpha_{e}}{1-\frac{4}{9} \pi N\left(2 \alpha_{o}+\alpha_{e}\right)}-\frac{1}{2}\right)\right]^{1 / 2},
$$

and then expand Eq. (6) into power series:

$$
n_{e} \approx \frac{3 \sqrt{2}}{4}+\frac{\sqrt{2} \pi N \alpha_{e}}{1-\frac{4}{9} \pi N\left(2 \alpha_{o}+\alpha_{e}\right)} .
$$

Similarly, $n_{o}$ can be expanded as

$$
n_{o} \approx \frac{3 \sqrt{2}}{4}+\frac{\sqrt{2} \pi N \alpha_{o}}{1-\frac{4}{9} \pi N\left(2 \alpha_{o}+\alpha_{e}\right)} .
$$

From the above equations, the wavelength and temperature effects of LC refractive indices are determined by $\alpha_{e}$ and $\alpha_{o}$. We need to find how $\alpha_{e}$ and $\alpha_{o}$ vary with wavelength and temperature.

Liquid crystal is a state of matter with physical properties lying between an isotropic liquid and a perfect crystal. Thus, its molecular differential polarizability $\alpha_{e}-\alpha_{o}$ is related to the crystalline state $\gamma_{e}-\gamma_{o}$ by the order parameter as $^{13}$

$$
\alpha_{e}-\alpha_{o}=S\left(\gamma_{e}-\gamma_{0}\right) \text {. }
$$

The average molecular polarizabilities $\langle\alpha\rangle$ and $\langle\gamma\rangle$ are related to their individual molecular polarizabilities as

$$
\begin{aligned}
& \langle\alpha\rangle=\frac{1}{3}\left(2 \alpha_{0}+\alpha_{e}\right), \\
& \langle\gamma\rangle=\frac{1}{3}\left(2 \gamma_{0}+\gamma_{e}\right) .
\end{aligned}
$$

Based on Eqs. (8)-(10), the individual molecular polarizabilities can be expressed as

$$
\begin{aligned}
& \gamma_{e}=\langle\alpha\rangle+\frac{2\left(\alpha_{e}-\alpha_{o}\right)}{3 S}, \\
& \gamma_{0}=\langle\alpha\rangle-\frac{\alpha_{e}-\alpha_{o}}{3 S}, \\
& \alpha_{o}=\langle\gamma\rangle-\frac{S}{3}\left(\gamma_{e}-\gamma_{0}\right), \\
& \alpha_{e}=\langle\gamma\rangle+\frac{2 S}{3}\left(\gamma_{e}-\gamma_{0}\right) .
\end{aligned}
$$

Substituting Eqs. (13) and (14) back to Eq. (9), we prove that:

$$
\langle\gamma\rangle=\langle\alpha\rangle
$$

That means the average molecular polarizabilities in the liquid crystal state and crystalline state are essentially equal. Plug Eqs. (13)-(15) back to Eqs. (7a) and (7b), we obtain

$$
n_{e} \approx \frac{3 \sqrt{2}}{4}+\frac{\sqrt{2} \pi N\langle\alpha\rangle}{1-\frac{4}{3} \pi N\langle\alpha\rangle}+\frac{\frac{2 \sqrt{2}}{3} \pi N S\left(\gamma_{e}-\gamma_{0}\right)}{1-\frac{4}{3} \pi N\langle\alpha\rangle}
$$




$$
n_{o} \approx \frac{3 \sqrt{2}}{4}+\frac{\sqrt{2} \pi N\langle\alpha\rangle}{1-\frac{4}{3} \pi N\langle\alpha\rangle}-\frac{\frac{\sqrt{2}}{3} \pi N S\left(\gamma_{e}-\gamma_{0}\right)}{1-\frac{4}{3} \pi N\langle\alpha\rangle}
$$

In Eqs. (16) and (17), the first term $3 \sqrt{2} / 4 \sim 1.0607$ is close to unity, the second term is weakly dependent on temperature through the molecular packing density $N$ and the average molecular polarizability $\langle\alpha\rangle$, and the third term is sensitive to the temperature as it depends on the order parameter. In a temperature not too close to the clearing temperature $\left(T_{c}\right)$, the order parameter can be approximated as ${ }^{14}$

$$
S=\left(1-T / T_{c}\right)^{\beta}
$$

where $\beta$ is a material parameter; it depends on the LC molecular structure. In an isotropic state, $S=0$ and the third term vanish. Under such a circumstance, $n_{e}=n_{o}=n_{i}$ with

$$
n_{i}(\lambda) \approx 1.0607+\frac{\sqrt{2} \pi N\langle\alpha\rangle}{1-\frac{4}{3} \pi N\langle\alpha\rangle} .
$$

For 5CB in the isotropic state, $n_{i} \sim 1.647$ at $\lambda=400 \mathrm{~nm}$ and $n_{i} \sim 1.580$ at $\lambda=800 \mathrm{~nm}^{7}$ From $\lambda=400$ to $800 \mathrm{~nm}$, the refractive index is only decreased by $\sim 4 \%$. That means in the isotropic state the refractive index is relatively insensitive to the wavelength. Substituting these numbers into Eq. (19), we find $0.247<\pi N\langle\alpha\rangle<0.267$, indicating that $\pi N\langle\alpha\rangle$ is not sensitive to the wavelength. This approximation should be applicable to other LC compounds as well. On the other hand, the temperature effect of $n_{i}$ is through the packing density $(N)$ variation, which is also very weak (for $5 \mathrm{CB}$, $\left.d n_{i} / d T \sim 4 \times 10^{-4}\right)$.

In the off-resonance region, the molecular polarizability $\left(\alpha_{e, o}\right)$ is related to the electronic transition wavelength $\lambda_{i}$ and the corresponding oscillator strength $\left(f_{e, o}\right)_{i}$ as ${ }^{15}$

$$
\alpha_{e, o}(\lambda) \approx \sum_{i}\left(f_{e, o}\right)_{i} \frac{\lambda^{2} \lambda_{i}^{2}}{\lambda^{2}-\lambda_{i}^{2}} .
$$

In Eq. (20), $\lambda_{i}$ represents the three electronic transition bands involved. In the off-resonance region where $\lambda \gg \lambda_{i}$, the three bands can be merged to a single band with resonance wavelength $\lambda *:^{4}$

$$
\gamma_{e, o} \propto Z f_{e, o}^{*} \frac{\lambda^{2} \lambda^{* 2}}{\lambda^{2}-\lambda^{* 2}} .
$$

Thus, the differential molecular polarizability can be rewritten as

$$
\gamma_{e}-\gamma_{o}=g Z\left(f_{e}^{*}-f_{o}^{*}\right) \frac{\lambda^{2} \lambda^{* 2}}{\lambda^{2}-\lambda^{* 2}},
$$

where $g$ is a proportionality constant, $Z$ is the number of active electrons per molecule, and $\left(f^{*}\right)$ is the effective oscillator strength at the mean resonance wavelength $\lambda^{*}$. Substituting Eqs. (19) and (22) back to Eq. (16), we obtain

$$
n_{e}(\lambda, T) \approx n_{i}(\lambda)+\frac{\frac{2 \sqrt{2}}{3} \pi g N Z S\left(f_{e}^{*}-f_{o}^{*}\right)}{1-\frac{4}{3} \pi N\langle\alpha\rangle} \frac{\lambda^{2} \lambda^{* 2}}{\lambda^{2}-\lambda^{* 2}} .
$$

As mentioned above, the (4/3) $\pi N\langle\alpha\rangle$ term in the denominator of Eq. (23) is insensitive to wavelength and temperature and, to the first order, can be treated as a constant. As a result, Eq. (23) can be further simplified as

$$
n_{e}(\lambda, T) \approx n_{i}(\lambda)+G S \frac{\lambda^{2} \lambda^{* 2}}{\lambda^{2}-\lambda^{2}} .
$$

Similarly, $n_{o}$ (and, hereafter, $\Delta n=n_{e}-n_{o}$ ) can be derived through the same process. Results are

$$
\begin{aligned}
& n_{o}(\lambda, T) \approx n_{i}(\lambda)-\frac{G S}{2} \frac{\lambda^{2} \lambda^{* 2}}{\lambda^{2}-\lambda^{* 2}}, \\
& \Delta n(\lambda, T) \approx \frac{3 G S}{2} \frac{\lambda^{2} \lambda^{* 2}}{\lambda^{2}-\lambda^{* 2}},
\end{aligned}
$$

where

$$
G=\frac{2 \sqrt{2}}{3} \pi g N Z\left(f_{e}^{*}-f_{o}^{*}\right) /\left(1-\frac{4}{3} \pi N\langle\alpha\rangle\right),
$$

is a proportionality constant.

The temperature effect of $N$ and $f_{e}^{*}-f_{o}^{*}$ is much smaller than that of S. Thus, we can assume $G$ is insensitive to the temperature. Equation (24c) is identical to the single-band birefringence dispersion model, ${ }^{4}$ although the derivation procedures are not the same.

Although Eqs. (24a) and (24b) describe the wavelength and temperature effects of $n_{e}$ and $n_{o}$, they still involve the isotropic state refractive index $n_{i}(\lambda)$. In the isotropic state, $n_{i}(\lambda)$ can be expressed by the traditional Cauchy equation:

$$
n_{i}(\lambda)=A_{i}+\frac{B_{i}}{\lambda^{2}}+\frac{C_{i}}{\lambda^{4}}
$$

where $A_{i}, B_{i}$, and $C_{i}$ are the Cauchy coefficients for the isotropic state.

Figure 1 shows the fitting of Eq. (25) to the experimental results of $5 \mathrm{CB}$ at $T=36.1^{\circ} \mathrm{C}$, which is above the clearing temperature $\left(T_{c}\right)$ of $5 \mathrm{CB}\left(T_{c} \sim 35.3{ }^{\circ} \mathrm{C}\right)$. Indeed, a very good fitting is obtained. The three Cauchy coefficients extracted are: $A_{i}=1.5721, B_{i}=0.0021 \mu \mathrm{m}^{2}$, and $C_{i}$ $=0.0016 \mu \mathrm{m}^{4}$.

By substituting Eq. (25) into Eqs. (24a) and (24b), we obtain:

$$
\begin{aligned}
& n_{e}=A_{i}+\frac{B_{i}}{\lambda^{2}}+\frac{C_{i}}{\lambda^{4}}+G S \frac{\lambda^{2} \lambda^{* 2}}{\lambda^{2}-\lambda^{2}}, \\
& n_{o}=A_{i}+\frac{B_{i}}{\lambda^{2}}+\frac{C_{i}}{\lambda^{4}}-\frac{G S}{2} \frac{\lambda^{2} \lambda^{* 2}}{\lambda^{2}-\lambda^{2}} .
\end{aligned}
$$

In the off-resonance region where $\left(\lambda \gg \lambda^{*}\right)$, we can expand the right-hand terms of Eqs. (26a) and (26b) into a power series. Retaining the $\lambda^{-2}$ and $\lambda^{-4}$ terms, we derive the following extended Cauchy equations: 


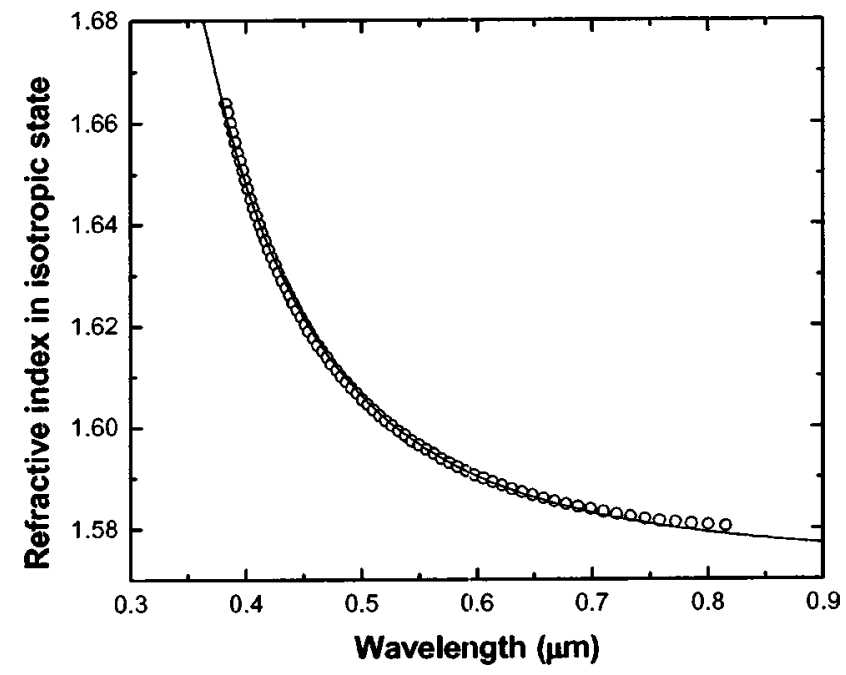

FIG. 1. Wavelength-dependent refractive index of 5CB in an isotropic state, $T=36.1^{\circ} \mathrm{C}$. Circles are experimental data and solid lines are fittings using Eq. (25). The fitting parameters are: $A_{i}=1.5721, B_{i}=0.0021 \mu \mathrm{m}^{2}$, and $C_{i}$ $=0.0016 \mu \mathrm{m}^{4}$.

$$
\begin{aligned}
& n_{e}=A_{e}+\frac{B_{e}}{\lambda^{2}}+\frac{C_{e}}{\lambda^{4}}, \\
& A_{e}=A_{i}+A_{e}^{\prime} S, \\
& B_{e}=B_{i}+B_{e}^{\prime} S, \\
& C_{e}=C_{i}+C_{e}^{\prime} S,
\end{aligned}
$$

where $A_{e}^{\prime}=G \lambda^{* 2}, B_{e}^{\prime}=G \lambda^{* 4}, C_{e}^{\prime}=G \lambda^{* 6}$, and $A_{i}, B_{i}$, and $C_{i}$ are the Cauchy coefficients of the LC in its isotropic state. By the same procedures, we find the Cauchy coefficients for $n_{o}$ :

$$
\begin{aligned}
& n_{o}=A_{o}+\frac{B_{o}}{\lambda^{2}}+\frac{C_{o}}{\lambda^{4}}, \\
& A_{o}=A_{i}-A_{o}^{\prime} S, \\
& B_{o}=B_{i}-B_{o}^{\prime} S, \\
& C_{o}=C_{i}-C_{o}^{\prime} S,
\end{aligned}
$$

where $A_{o}^{\prime}=G \lambda^{* 2} / 2, B_{o}^{\prime}=G \lambda^{* 4} / 2$, and $C_{o}^{\prime}=G \lambda^{* 6} / 2$.

Equations (27) and (28) give the complete temperature and wavelength dependence of the liquid crystal refractive indices. Equations (27) and (28) can be applied to LC mixtures as well. The $n_{e}$ and $n_{o}$ of each compound in the mixture share the same forms as Eqs. (27a) and (28a) except for the different Cauchy coefficients. These individual equations can be superimposed and still lead to the same Cauchy form. Thus, the extended Cauchy equations can be applied to single compounds and mixtures.

As shown in Eqs. (27a) and (28a), at a given temperature both $n_{e}$ and $n_{o}$ decrease as the wavelength increases. In the long wavelength region $(\lambda>1 \mu \mathrm{m})$, the contributions of the $B$ and $C$ terms become negligible. Under these conditions, $n_{e} \rightarrow A_{e}$ and $n_{o} \rightarrow A_{o}$; they are independent of wavelength. This result is consistent with many experimental evidences.

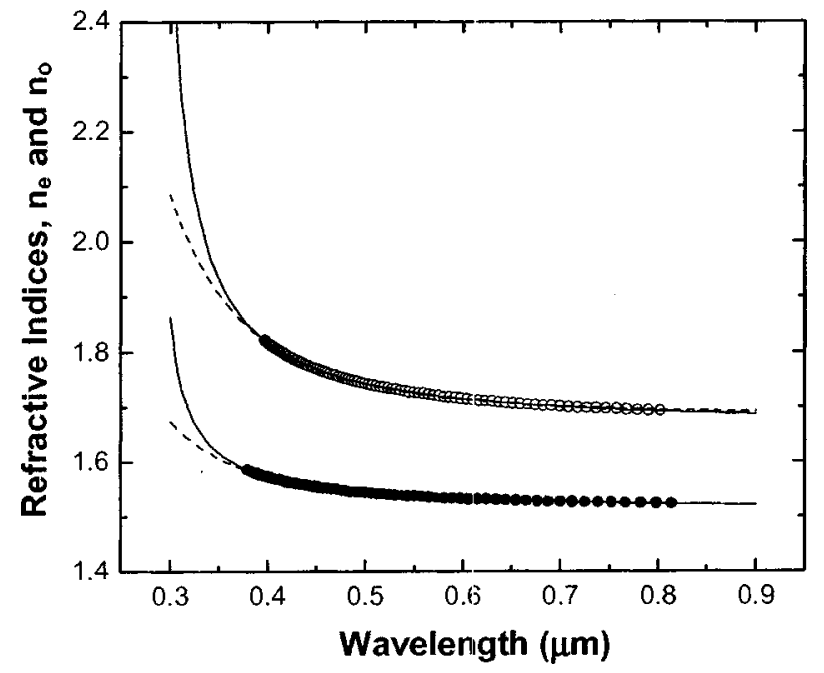

FIG. 2. Wavelength-dependent refractive indices of $5 \mathrm{CB}$ at $T=25.1^{\circ} \mathrm{C}$. Open and closed circles are experimental data for $n_{e}$ and $n_{o}$, respectively. Solid line represents the three-band model and dashed lines are for the extended Cauchy model. The fitting parameters are listed in Table I.

Equations (27) and (28) predict that if we plot the Cauchy coefficients as a function of the order parameter, straight lines with positive slopes for $n_{e}$ and negative slopes for $n_{o}$ should be found. Moreover, each corresponding parameter for $n_{e}$ and $n_{o}$ (e.g., $A_{e}$ and $A_{o}$ ) will intersect at $S$ $=0$, and the intersections are $A_{i}, B_{i}$, and $C_{i}$; the Cauchy's coefficients in the isotropic phase.

\section{RESULTS AND DISCUSSIONS}

The extended Cauchy model we derive here has three fitting parameters. To validate this model, we need to have at least half a dozen data points. From the literature, we found that 5CB has the most complete experimental refractive index data. ${ }^{7}$ At a given temperature, there are more than 50 refractive index data measured spanning in the 400-800 nm spectral range.

\section{A. Comparisons of models}

Figure 2 depicts the fittings of the experimental data of $5 \mathrm{CB}$ using the three-band model (solid lines) and the extended Cauchy equations (dashed lines). The fitting parameters are listed in Table I. In the visible and near-infrared regions, both models give excellent fits to the experimental data. In the UV region, the deviation between these two models becomes more apparent. The three-band model con-

TABLE I. Fitting parameters for the three-band model and the extended Cauchy equations. LC: $5 \mathrm{CB}$ at $T=25.1^{\circ} \mathrm{C}$. The units of Cauchy's B and C coefficients are $\mu \mathrm{m}^{2}$ and $\mu \mathrm{m}^{4}$, respectively.

\begin{tabular}{ccccccc}
\hline \hline Model & \multicolumn{3}{c}{$n_{e}$} & & $n_{o}$ & \\
\hline $\begin{array}{c}\text { Three-band } \\
\text { model }\end{array}$ & $n_{0 e}$ & $g_{1 e}$ & $g_{2 e}$ & $n_{0 o}$ & $g_{1 o}$ & $g_{2 o}$ \\
& 0.4618 & 2.1042 & 1.4413 & 0.4202 & 1.2286 & 0.4934 \\
Cauchy & $A_{e}$ & $B_{e}$ & $C_{e}$ & $A_{o}$ & $B_{o}$ & $C_{o}$ \\
model & 1.6795 & 0.0048 & 0.0027 & 1.5187 & 0.0016 & 0.0011 \\
\hline \hline
\end{tabular}




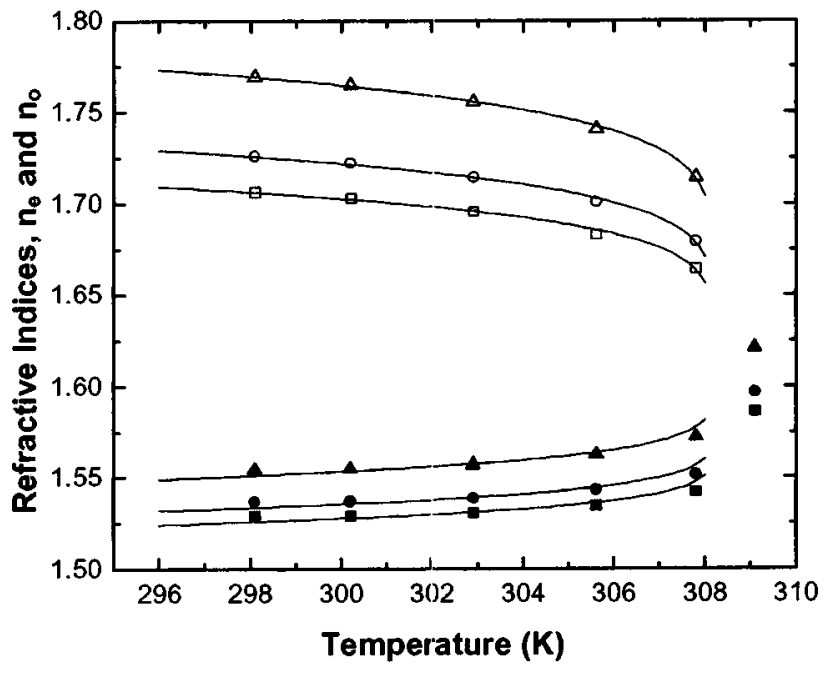

FIG. 3. Temperature-dependent refractive indices of 5CB. Open and filled triangles, circles, and squares are experimental data for $n_{e}$ and $n_{o}$ at $\lambda$ $=450,550$, and $650 \mathrm{~nm}$, respectively. Measurement temperatures are 25.1, $27.2,29.9,32.6,34.8$, and $36.1^{\circ} \mathrm{C} . T_{c}=35.3{ }^{\circ} \mathrm{C}$. Solid lines are fitting curves using Eqs. (29a) and (29b).

siders the resonance effect, but the extended Cauchy model does not. Thus, in the near-resonance region the results from the three-band model are more accurate.

\section{B. Temperature effect}

From Eqs. (24a) and (24b), at a given wavelength, the refractive indices have the following forms:

$$
\begin{aligned}
& n_{e}(T) \approx n_{i}+G^{\prime}\left(1-\frac{T}{T_{c}}\right)^{\beta}, \\
& n_{o}(T) \approx n_{i}-\frac{G^{\prime}}{2}\left(1-\frac{T}{T_{c}}\right)^{\beta},
\end{aligned}
$$

where $n_{i}$ is the refractive index in the isotropic state and $G^{\prime}=G\left[\lambda^{2} \lambda^{* 2} /\left(\lambda^{2}-\lambda^{* 2}\right)\right]$ and $\beta$ are fitting parameters. For $5 \mathrm{CB}$, the experimental refractive index data at $T=25.1,27.2$, 29.9, 32.6, 34.8, and $36.1^{\circ} \mathrm{C}$ are available in the $\lambda$ $=400-800 \mathrm{~nm}$ range. To illustrate the calculation methods, we selected the data at three primary colors $(\lambda=450,550$, and $650 \mathrm{~nm}$ ) used for display applications.

Figure 3 plots the temperature-dependent refractive indices of 5CB at $\lambda=450$ (triangles), 550 (circles), and $650 \mathrm{~nm}$ (squares). The solid lines are fitting curves using parameters listed in Table II. The fitting results are quite satisfactory. As
TABLE II. Parameters obtained by fitting temperature-dependent $n_{e}$ and $n_{o}$ data of 5CB using Eqs. (29a) and (29b).

\begin{tabular}{ccccccc}
\hline \hline \multirow{2}{*}{$\lambda(\mathrm{nm})$} & & \multicolumn{2}{c}{$n_{e}$} & & \multicolumn{2}{c}{$n_{o}$} \\
\cline { 3 - 4 } \cline { 5 - 6 } & $n_{i}$ & $G^{\prime}$ & $\beta$ & & $G^{\prime}$ & $\beta$ \\
\hline 450 & 1.6209 & 0.2440 & 0.1461 & & 0.2298 & 0.1461 \\
550 & 1.5965 & 0.2092 & 0.1414 & & 0.2042 & 0.1414 \\
650 & 1.5865 & 0.1914 & 0.1375 & & 0.1942 & 0.1375 \\
\hline \hline
\end{tabular}

the temperature increases, $n_{e}$ decreases gradually while $n_{o}$ increases slightly. At a given temperature, both $n_{e}$ and $n_{o}$ increase as the wavelength decreases.

It is noteworthy that Eqs. (29a) and (29b) are interrelated. Although each equation has two adjustable parameters ( $G^{\prime}$ and $\beta$; provided that $n_{i}$ is known), these two parameters have to fit both $n_{e}$ and $n_{o}$ simultaneously. During the fitting process, we first obtained $G^{\prime}$ and $\beta$ for each wavelength by fitting the temperature-dependent $n_{e}$ of $5 \mathrm{CB}$ using Eq. (29a). We then fixed the $\beta$ value and fitted the $n_{o}$ data through Eq. (29b) by only using $G^{\prime}$ as a variable parameter. For the RGB wavelengths studied, the obtained $G^{\prime}$ values for $n_{e}$ and $n_{o}$ agree within 5\%. From Table II, $G^{\prime}$ decreases as the wavelength increases. The same trend holds for both $n_{e}$ and $n_{o}$. In principle, the $\beta$ exponent should not depend on the wavelength employed. From Table II, we find $\beta=0.142$ \pm 0.004 , the variation is within $\pm 3 \%$.

\section{Correlations between Cauchy coefficients}

According to Eqs. (27) and (28), the Cauchy coefficients for both $n_{e}$ and $n_{o}$ are linearly proportional to the order parameter $(S)$. If we plot the Cauchy coefficients as a function of $S$, then straight lines with positive and negative slopes for $n_{e}$ and $n_{o}$, respectively, should be obtained. The interceptions at $S=0$ lead to the Cauchy coefficients at the isotropic state.

To validate these predictions, we used the experimental data of $5 \mathrm{CB}$. The order parameter described in Eq. (18) is determined by the clearing temperature $T_{c}$ and $\beta$ exponent. The clearing temperature of $5 \mathrm{CB}$ is $308.3 \mathrm{~K}$. Therefore, by fitting the temperature-dependent birefringence data at a given wavelength we can obtain $\beta$ easily. The $\beta$ value we found is 0.142 (as listed in Table II). Using this $\beta$ value, we can calculate the order parameter at the specific measurement temperatures. In the mean time, by fitting the experimental data for $n_{e}$ and $n_{o}$ using the extended Cauchy equations, we

TABLE III. Cauchy coefficients and order parameters of 5CB. $T_{c}=308.3 \mathrm{~K}$ and $\beta=0.142$.

\begin{tabular}{cccccccr}
\hline \hline$T(\mathrm{~K})$ & $T / T_{c}$ & $S$ & $A_{e}$ & $B_{e}$ & $C_{e}$ & $A_{o}$ & $B_{o}$ \\
\hline 298.1 & 0.96723 & 0.61546 & 1.67946 & 0.00482 & 0.00273 & 1.51866 & 0.00163 \\
300.2 & 0.97404 & 0.59542 & 1.67649 & 0.00489 & 0.00266 & 1.51864 & 0.00163 \\
302.9 & 0.98280 & 0.56161 & 1.67030 & 0.00462 & 0.00257 & 1.52012 & 0.00158 \\
305.6 & 0.99156 & 0.507594 & 1.65866 & 0.00440 & 0.00248 & 1.52369 & 0.00174 \\
307.8 & 0.99870 & 0.389115 & 1.64304 & 0.00380 & 0.00217 & 1.53028 & 0.00183 \\
309.1 & 1.00292 & 0 & 1.57209 & 0.00210 & 0.00161 & 1.57209 & 0.00116 \\
\hline \hline
\end{tabular}




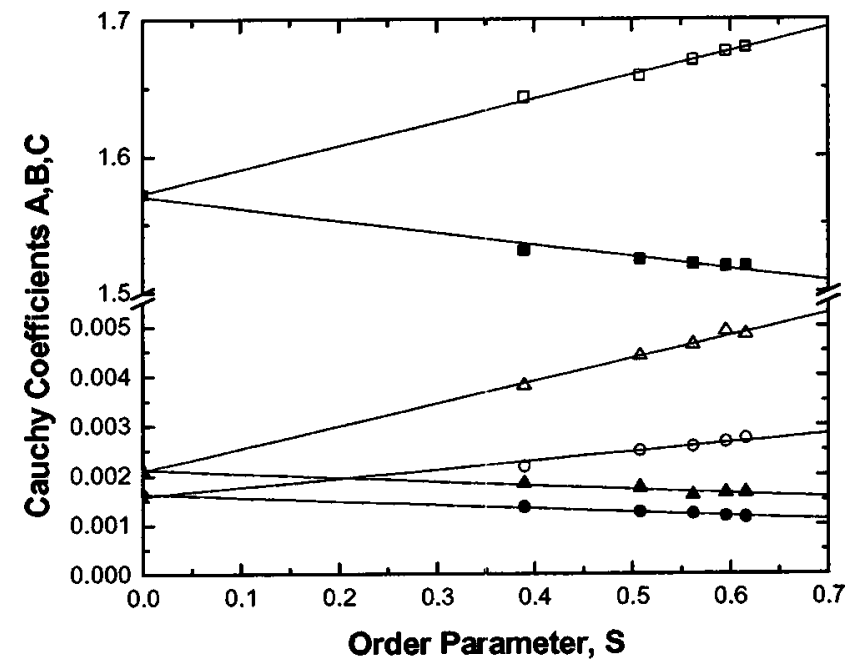

FIG. 4. Order parameter dependent Cauchy coefficients. Squares, triangles, and circles are the $A B C$ Cauchy coefficients obtained by fitting the $5 C B$ data at six different temperatures. Open and filled squares, triangles, and circles are for $\left[A_{e}, A_{o}\right]\left[B_{e}, B_{o}\right]$, and $\left[C_{e}, C_{o}\right]$, respectively. Solid lines are fitting results.

could extract the corresponding Cauchy coefficients. The results are listed in Table III.

Figure 4 shows the order parameter dependent Cauchy coefficients $A_{e, o}$ (squares), $B_{e, o}$ (triangles), and $C_{e, o}$ (circles) for 5CB. Indeed, straight lines are found. The $A_{e}, B_{e}$, and $C_{e}$ coefficients have positive slopes while $A_{o}, B_{o}$, and $C_{o}$ have negative slopes. The slope $\left(A_{e}^{\prime}, A_{o}^{\prime}\right.$, etc.) of each line is listed in Tables IV for $n_{e}$ and $n_{o}$, respectively. The corresponding line pairs $\left(A_{e}, A_{o}\right),\left(B_{e}, B_{o}\right)$, and $\left(C_{e}, C_{o}\right)$ intersect at $S=0$. The interceptions agree quite well with the Cauchy coefficients $A_{i}, B_{i}$, and $C_{i}$ obtained from the isotropic phase.

Based on the Cauchy coefficients listed in Table IV, the birefringence of $5 \mathrm{CB}$ can be calculated at any desired wavelength and temperature. This feature is particularly important for the elevated temperature operation of liquid crystal devices.

\section{CONCLUSIONS}

We have developed extended Cauchy equations for describing the wavelength- and temperature-dependent refrac-
TABLE IV. Parameters $A_{e, o}^{\prime}, B_{e, o}^{\prime}, C_{e, o}^{\prime}, A_{i}, B_{i}$, and $C_{i}$ obtained from the slopes and interceptions in Fig. 4.

\begin{tabular}{ccccccc}
\hline \hline $5 \mathrm{CB}$ & \multicolumn{6}{c}{ Cauchy coefficients } \\
\hline \multirow{2}{*}{$n_{e}$} & $A_{e}^{\prime}$ & $A_{i}$ & $B_{e}^{\prime}$ & $B_{i}$ & $C_{e}^{\prime}$ & $C_{i}$ \\
& 0.1738 & 1.5721 & 0.0045 & 0.0021 & 0.0018 & 0.0016 \\
$n_{o}$ & $A_{o}^{\prime}$ & $A_{i}$ & $B_{o}^{\prime}$ & $B_{i}$ & $C_{o}^{\prime}$ & $C_{i}$ \\
& 0.0885 & 1.5721 & 0.0008 & 0.0021 & 0.0007 & 0.0016 \\
\hline \hline
\end{tabular}

tive indices of liquid crystal compounds and mixtures. The model fits well with the experimental results of 5CB. This model is particularly useful for fitting the experimental data of liquid crystal mixtures where the three-band model fails. However, in the near-resonance region, the extended Cauchy model has less accuracy than that of the three-band model.

\section{ACKNOWLEDGMENTS}

The authors are indebted to AFOSR for financial support under Contract No. F49620-01-1-0377.

${ }^{1}$ H. E. Neugebauer, Can. J. Phys. 32, 1 (1954).

${ }^{2}$ M. F. Vuks, Opt. Spektrosk. 20, 644 (1966).

${ }^{3}$ W. H. de Jeu, Physical Properties of Liquid Crystalline Materials (Gordon and Breach, New York, 1980), Chap. 4.

${ }^{4}$ S. T. Wu, Phys. Rev. A 33, 1270 (1986).

${ }^{5}$ S. T. Wu, J. Appl. Phys. 69, 2080 (1991).

${ }^{6}$ I. Abdulhalim, Mol. Cryst. Liq. Cryst. 197, 103 (1991).

${ }^{7}$ S. T. Wu, C. S. Wu, M. Warenghem, and M. Ismaili, Opt. Eng. (Bellingham) 32, 1775 (1993).

${ }^{8}$ I. C. Khoo and S. T. Wu, Optics and Nonlinear Optics of Liquid Crystals (World Scientific, Singapore, 1993).

${ }^{9}$ E. M. Averyanov, J. Opt. Technol. 64, 417 (1997).

${ }^{10} \mathrm{M}$. Born and E. Wolf, Principle of Optics, 6th ed. (Pergamon, New York, 1980).

${ }^{11}$ H. Mada and S. Kobayashi, Mol. Cryst. Liq. Cryst. 33, 47 (1976).

${ }^{12}$ L. Pohl and U. Finkenzeller, in Liquid Crystals: Applications and Uses, edited by B. Bahadur (World Scientific, Singapore, 1990), Vol. 1, Chap. 4.

${ }^{13}$ L. M. Blinov, Electro-Optical and Magneto-Optical Properties of Liquid Crystals (Wiley, New York, 1983), Chap. 2.

${ }^{14}$ I. Haller, Prog. Solid State Chem. 10, 103 (1975).

${ }^{15}$ A. D. Buckingham, in Molecular Electro-Optics, edited by C. T. O'Konski (Dekker, New York, 1976), Chap. 2. 